\title{
Preparation and evaluation of the influence of modified fiber flour wood on the properties of the fresh condition of cement-based mortars
}

\author{
Aloulou Fadhel $^{1}$ - Alila Sabrine ${ }^{2}$ \\ Received: 7 December 2017 / Accepted: 4 October 2018 / Published online: 12 October 2018 \\ (c) The Author(s) 2018
}

\begin{abstract}
Wood fibers were used as fillers in the partial cement matrix by replacing the cement to a content of $1 \%$ by weight of cement. The effect of wood fibers on porosity and compressive strength has been studied. The results obtained show an improvement in the compressive strength of more than $40 \%$ with $1 \%$ by weight of wood fibers. The addition of wood fibers shows a good pore reduction, and the best result was obtained with the emulsion of a mixture incorporating $1 \%$ by weight of wood fibers in the presence of an anionic surfactant (SDBS). The degree of hydration of the cement increases with the wood fibers. This property was confirmed by Fourier transform infrared spectroscopy. These analyzes revealed that the presence of wood fibers generates and promotes the hydration of the cement, producing more calcium silicate gel and portlandite, which affects the compressive strength which gives a strong improvement.
\end{abstract}

Keywords Wood · Fiber · Portland cement composite · Degree of cement hydration · Mechanical properties · Microstructural properties

$\begin{array}{ll}\text { Abbreviations } & \\ \mathrm{C}-\mathrm{S}-\mathrm{H} & \text { Calcium silicate hydrates } \\ \mathrm{NaOH} & \text { Sodium hydroxide } \\ \text { PC } & \text { Ordinary Portland cement NT } \\ & \text { Sodi:1983 } \\ \text { SDBS } & \text { Ehylenediaminetetraacetic acid } \\ \text { EDTA } & \text { Potassium bromide } \\ \mathrm{KBr} & \text { Tetramethylsilane } \\ \mathrm{TMS} & \text { Critical micellar concentration } \\ \mathrm{CMC} & \text { Wood fiber treated with a soda } \\ \text { Wood-NaOH } & \text { solution }\end{array}$

Aloulou Fadhel

alouloufadhel@gmail.com

Alila Sabrine

sabrine_alila@yahoo.fr

1 LabEM-LR11ES34-Laboratory of Energy and Materials, University of Sousse, Sousse, Tunisia

2 LMSE-Laboratoire Sciences DES Matériaux Et Environnement, University of Sfax, Sfax, Tunisia

$\begin{array}{ll}\text { Wood-NaOH-EDTA } & \begin{array}{l}\text { Wood fiber treated with sodium } \\ \text { hydroxide solution and EDTA }\end{array} \\ & \text { solution } \\ \text { Wood fiber modified by esterifica- } \\ \text { Wood modified }\end{array} \quad \begin{aligned} & \text { tion with acetic anhydride }\end{aligned}$

\section{Introduction}

The use of the plant fibers represents a new stake in front of problems facing the recycling of composites strengthened with synthetic fibers [1]. It appears from the literature and in touch with this subject that even if the concept is not new, it is not applied, at the moment, to the industrial scale and the majority of the related products remain unexploited, with the exceptions of sawdust which are used as fuels [1].

The concept of strengthening the cement matrix with plant fibers was developed as a potential substitute for asbestos fibers in 1940 [2]. The use of plant fibers in the reinforcement of building materials is a relatively old field, noting for example the impregnation as horse hair in filling mortars or the manufacture of earth blocks reinforced with straw. In cement-based composites, cellulose fibers have been used as pulp, short fibers, long fibers or fabrics [3, 4]. However, the traditional methods used did not obey any 
technological development and scientific analysis until 1970, when the first work was done to replace asbestos fibers in prefabricated profiled fiber cement elements [5] composites, cellulose fiber has been used as pulp, short fibers, long fibers or fabrics [3, 4].

Rice et al. [6] studied the possibility of substituting the synthetic fibers by natural fibers such as the coconut, the sugar cane, the bugs and the fibers of banana' trees. He showed that the epoxy strengthening of concretes of polymer by the fiber of coconut slightly more than the others. Surface treatment of the cellulose fibers with various coupling agents, surface the durability of fiber and fiber-matrix interaction was reported in several studies. Ceylon coupling agents were found to be effective in modifying the natural fiber-matrix interface; silone treatment of cellulose fibers changes the surface topography, surface chemical structure, and thermal degradation of cellulose fibers [7].

Reinforcement of mineral binders (cement, concrete or gypsum) has been a major concern for several decades. Efforts have therefore been made to replace the usual reinforcing agents such as glass fibers or asbestos fibers with organic agents (extracts of biomass), such as sisal, kraft or cellulosic fibers [8]. To stabilize the plant fiber, three categories of treatment can be mentioned in the bibliography: thermal, physical and chemical.

Govin et al. [9] used a method called "roasting" for the treatment of fibers. This technique therefore consists of heating the wood fibers under an inert atmosphere up to $280{ }^{\circ} \mathrm{C}$ for a relatively long time to modify its structure. This process made it possible to limit the degradation of the fibers, so it kept its mechanical properties to a minimum value. Chemical treatment is the most studied technique worldwide; it consists of replacing the hydroxyl groups, highly hydrophilic and responsible for the swelling of the wood, by hydrophobic groups [10]. Several studies have shown the importance of treating plant fibers with chemical solutions to increase the adhesion between the fiber and the matrix and consequently to improve the mechanical properties. Toledo et al. [11] immersed sisal and coconut in three solutions of different $\mathrm{pH}$ values; first, treated the fibers in water at $\mathrm{pH}=8.3$ (weakly basic medium), then in a lime solution $\mathrm{Ca}(\mathrm{OH})_{2}$ at $\mathrm{pH}=12$ and finally in a solution of $\mathrm{NaOH}$ soda at $\mathrm{pH}=11$. The purpose of this type of treatment is to determine the impact of $\mathrm{pH}$ and the effect of calcium ions on the outer wall of the plant fibers after immersion.

Baley et al. [12, 13] studied the impact of different chemical treatments (soda, acetic anhydride, formic acid, EDTA, lime saturated water and a polyethylene solution) on the bond between linen fibers and polyester resin and compared them to the system glass-polyester. The interfacial characterization was carried out by removing the unit fiber from a micro drop of this thermosetting matrix. The interpretation of the results thus obtained has shown that the proposed treatments lead to a marked increase in the fiber-liner-polyester bond. The treatment with EDTA leads to an increase in the rupture stress of the composites. On the other hand, the influence of the two other treatments is low on the mechanical properties.

HM Saleh et al. [14-17] show that the cementation of 3\% of biological waste generated during phytoremediation using the dried aquatic plant Veronica anagallis-aquatica gave a satisfactory compressive strength of solidified material resulting from more than $13 \mathrm{MPa}$. The high hardness value exceeded $25 \mathrm{MPa}$ and was obtained in samples hardened in seawater or groundwater due to pore-sealing mineral salts. In another work, SB Eskander and HM Saleh [18-23] have studied the effect of incorporation the spinning wastes in cement and mortars, the performance of aging cement-polymer composite immobilizing borate waste simulates during flooding scenarios, the leaching behavior in the natural cement-clay composite incorporating a truly spent radioactive liquid scintillator.

In previous studies, our groups have shown that the adsorption of cationic surfactant on bleached cellulose fibers greatly enhances the aptitude of the substrate to uptake dissolved organic compounds from aqueous media [24-28]. The improved solute adsorption was ascribed to the accumulation of the organic solutes within the aggregated domains formed by the self-assembly of surfactant monomers at the cellulose/water interface. However, the desorption of the surfactant molecules from the cellulose surface impeded the regeneration of the substrate by extraction of the trapped solute once it is exhausted. Then, a chemical grafting of hydrocarbon structures that mimics the aggregated domains generated by the adsorbed surfactant molecules was accomplished [29]. The aptitude of the ensued modified substrate to uptake dissolved organic solute in aqueous media has been investigated in a batch and in continuous operations.

In this condition, the main environmental benefits such as biodegradability and renewal will be reduced. The purpose of this study was to evaluate the effect of treating wood fibers with anhydride and polymers derived from renewable resources, on the properties of wood fiber and cement-based composite. Water absorption and mechanical testing were done on wood fiber. Extraction tests were performed to evaluate the interface link. For the characterization of composites reinforced with $1 \%$ of wood fibers, compression tests were carried out.

In this study, the effect of the addition of $1 \%$ wood fiber on the mechanical and microstructural properties of cement paste composites was studied:

- Integration of wood fibers as a reinforcing agent in polymer-based composite materials: wood fibers form a rigid network, resulting from strong interactions between nanoparticles, is supposed to be governed by mechanical percolation mechanisms. 
- The addition of wood fibers improves the bending strength, the energy absorption of the cement paste, an increase in the cumulative heat of hydration and the degree of hydration of the cement of the reinforced wood fibers.

- The increase in the degree of hydration of the cement can be explained by the steric stabilization, which is responsible for the dispersion of the cement particles and by the effect of wood fibers which provide a channel for the transport of the water through the ring of hydration products (i.e., high density $\mathrm{CSH}$ ) to non-hydrated cement particles and improve hydration.

In this work, we continue our research regarding the potential use of modified wood fiber substrate as a new composite material with the cement matrix strengthened with vegetables fibers.

\section{Materials and methods}

\section{Wood fibers}

The wood fibers were recovered from industrial waste, the fibers were washed in hot water $\left(50^{\circ} \mathrm{C}\right)$ to remove surface residues, such as a mucilage extraction process, and cut to the length of $40 \mu \mathrm{m}$.

\section{Cement materials}

The cement used in the present study is the locally manufactured Portland cement (PC). This cement was manufactured according to Tunisian Standard Specifications NT 47-01:1983 [30] and the European standard EN 197-1:2000 [31]. The chemical composition and the physical characteristics of this cement are shown in Table 1.

\section{Adjuvant}

The additive used in our work is a commercial, analytical grade anionic surfactant were used as received, namely sodium dodecyl benzene sulfonate (SDBS). The critical micellar concentration $(\mathrm{CMC})$ in deionized water $\left(25^{\circ} \mathrm{C}\right)$ determined by conductimetry is $1.1 \times 10^{-3} \mathrm{~mol} \mathrm{~L}^{-1}$.

\section{FTIR analysis}

The Fourier transform infrared (FTIR) spectroscopy was used to analyze the composition change of the treated sisal. FTIR spectrum was obtained from $\mathrm{KBr}$ pellets with a Perkin Elmer spectrometer used in transmission mode with a resolution of $2 \mathrm{~cm}^{-1}$ in the range of $400-4000 \mathrm{~cm}^{-1}$.

\section{CP/MAS ${ }^{13} \mathrm{C}$ solid state NMR}

Cross polarization/magic angle spinning $(\mathrm{CP} / \mathrm{MAS}){ }^{13} \mathrm{C}$ solid state NMR experiments were performed with Bruker 300 spectrometer operating at a ${ }^{13} \mathrm{C}$ frequency of $75 \mathrm{MHz}$. the contact time for $\mathrm{CP}$ was $1 \mathrm{~ms}$ and the delay time for acquisitions was $5 \mathrm{~s}$. Chemical shifts were referred to tetramethylsilane (TMS).

\section{Contact angle}

The measure of the angle of contact is a technique bound to the capacity of a liquid in Spread out on a surface by wet ability. The principle of this characterization thus consists to measure the angle enter the tangent of the profile of a drop of the liquid put down on the substratum, and the surface of the substratum. She allows measuring the energy of surface of the liquid or of solid. In our work, we used the method of the angle of contact to deduct hydrophilic or hydrophobic character of the surface of the sample. In our case, we used the water, polar solvent, as liquid of measure of angle of contact, this liquid allows to deduct the hydrophobic character (short angle, low energy of surface) or hydrophilic (small angle, big energy of surface) from the surface. The contact angle used in this work device lead by a fast Pulnix TM 6701AN camera (capable of delivering 200 images from 100 to 320 pixels s${ }^{-1}$ ) is used to track the evolution of the shape of the drop every $5 \mathrm{~ms}$. The data is then processed directly in a computerized manner by means of a specific image processing program which makes it possible to follow the evolution of the contact angle as a function of the measurement time.

\section{Experimental approaches}

\section{Treatment processing by the soda}

This treatment processing was realized by the putting of wood fibers in an alkaline solution from the dissolution of $4 \mathrm{~g}$ of pastilles of hydroxide of sodium $\mathrm{NaOH}$ in $96 \mathrm{~cm}^{3}$ of the distilled water (the concentration of this solution is thus mass $4 \%$ ) during $20 \mathrm{~min}$. Then, these fibers were filtered and rinsed in big quantity of water to eliminate the excess of $\mathrm{NaOH}$, the last wash is made with some water containing a little acetic acid before they undergo an air drying (Fig. 1a).The objective of this treatment processing, which is relatively classic, is to clean
Table 1 Chemical composition and physical characteristics of Portland cement (wt\%)

\begin{tabular}{llllllllll}
\hline Material & $\mathrm{SiO}_{2}$ & $\mathrm{Al}_{2} \mathrm{O}_{3}$ & $\mathrm{Fe}_{2} \mathrm{O}_{3}$ & $\mathrm{CaO}$ & $\mathrm{MgO}$ & $\mathrm{SO}_{3}$ & $\mathrm{Na}_{2} \mathrm{O}$ & $\mathrm{K}_{2} \mathrm{O}$ & Loss on ignition \\
\hline Cement & 19.4 & 4.8 & 3.6 & 63.7 & 1.9 & 2.7 & 0.2 & 0.8 & 2.4 \\
\hline
\end{tabular}


the surface of the fibers. Indeed, the soda allows removing partially the amorphous structures which exist on the surface of fibers such as fats and wax. The dumping of the vegetable fibers in an alkaline environment showed a big efficiency to produce solid fibers containing a low content lignin, and a good separation of fibers. However, no direct measure of the decrease of the rate of lignin was made. The lignin which is relatively stiff but incompatible chemically with the cement can reduce the surface of connection between fiber and matrix.

\section{Treatment processing by EDTA}

The vegetable fibers so handled by the soda require a treatment processing with a having complexes agent, it is about some ethylenediaminetetraacetic acid (EDTA). This stage of treatment processing was realized by the dumping of wood fibers in a containing aqueous solution $1 \mathrm{~g}$ of EDTA in $99 \mathrm{~cm}^{3}$ of the water (of concentration 1\%). The treatment processing lasts $10 \mathrm{~min}$. The use of the EDTA $\left(\mathrm{C}_{10} \mathrm{H}_{16} \mathrm{~N}_{2} \mathrm{O}_{2}\right)$ aims at preventing the fixation (binding) of the ions calcium $\mathrm{Ca}^{2+}$ on the surface of the fibers, exceptionally by pectin. Indeed, the molecules of EDTA train a very stable complex with the calcium, and as a consequence contribute to the destruction of the complex pectin-calcium on the surface of the fibers. The treatment processing with the EDTA allows increasing the constraint in the break of realizing mortars, while fixing the minimum of calcium to the surface of the fibers, because the existence of the latter of surface influences the constraint in the break. The treatment processing in the EDTA seems to lead at the beginning of separation of fibers some of the others, by degrading the amorphous constituents of fibers (Fig. 1b).

\section{Treatment by esterification}

This study involves the grafting of linear alkyl chains with different lengths of wood fibers by esterification using fatty acid anhydride. We exploited the hydroxyl functions of wood-fibers to condense them by acylation with $\mathrm{C} 12$ anhydride at different levels. Due to the low reactivity of the carboxylic acid functions present in the wood fibers with respect to the esterification reactions, we have activated this function by converting it into a much more reactive anhydride than the method of elaboration and characterization of the composites studied [27]. By the following three stages: preparation of materials (cement, fibers and water), elaboration of the studied composites and preparation of the molds. The implementation of composites is done using our methods; we tried to make three forms of samples shown in Fig. 1c. After homogenization of the fresh material, it is put into place in molds. $24 \mathrm{~h}$ later, the samples were removed and left to continue drying in ambient air for 10 days.

\section{Formulation of the cementitious product}

Cylindrical blocks of mortar were prepared by different ratios cement/wood fiber with or without volume variation. The prepared mortar paste was poured into cylindrical plastic molds $70 \mathrm{~mm}$ in height and $35 \mathrm{~mm}$ in diameter for each test composition (Table 2). This paste was compacted manually for a fixed time and then tightly closed with plastic covers. The specimens were fabricated and processed for laboratory hardening and curing at a temperature of $25 \pm 5^{\circ} \mathrm{C}$ in their wet conditions for 28 days of curing according to NT 47-30: 1991 [32].

\section{Results and discussion}

\section{Characterization of modified fibers}

\section{Analysis by infrared spectroscopy FTIR}

The wood fiber modified in this study obtained by heterogeneous esterification with $\mathrm{C} 12$ anhydride. The modified wood fiber was analyzed by FTIR and solid state NMR.
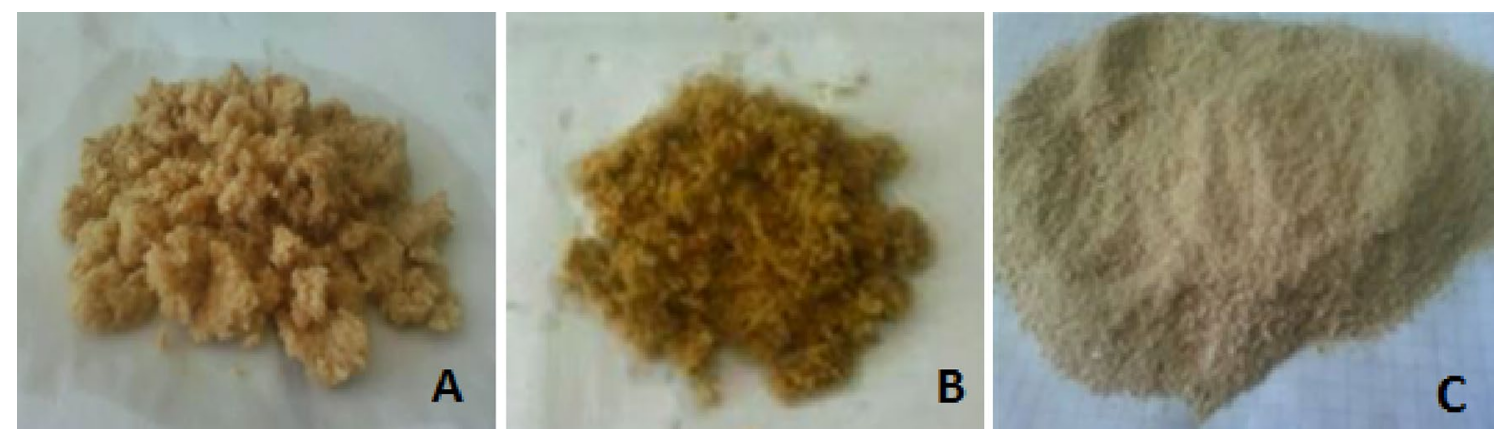

Fig. 1 a Treatment by the soda, $\mathbf{b}$ treatment by EDTA, $\mathbf{c}$ treatment by esterification 
Table 2 Shapes and dimensions of specimens

\begin{tabular}{|l|c|c|l|}
\hline Shapes & Picture & Sizing & Manufacturing \\
\hline Pellets & & $\begin{array}{c}\Phi=3.3 \mathrm{~cm} \\
\mathrm{~h}=1.5 \mathrm{~cm}\end{array}$ & $\begin{array}{l}\text { Absorbance } \\
\text { measuring }\end{array}$ \\
\hline Cylinder & & & \\
\hline
\end{tabular}

FTIR spectra, shown in Fig. 2, exhibits the presence of an ester carboxyl absorption band at $1732 \mathrm{~cm}^{-1}$, methylene peak at $2855 \mathrm{~cm}^{-1}$ and $1450 \mathrm{~cm}^{-1}$. The other characteristic peaks of acyl moiety are hidden by the overlapping of wood fiber bundles.

\section{Analysis by CP/MAS ${ }^{13} \mathrm{C}$ solid state NMR}

The NMR spectra of fiber wood and fiber wood modified are shown in Fig. 3. In the spectrum of fiber wood, all signals, i.e., those at $104.7 \mathrm{ppm}(\mathrm{C}-1), 89.8 \mathrm{ppm}$ (C-4 of crystalline cellulose), $74.7 \mathrm{ppm}(\mathrm{C}-5), 72 \mathrm{ppm}(\mathrm{C}-2$ and $\mathrm{C}-3)$, and $69.5 \mathrm{ppm}$ (C-6 of crystalline cellulose) [33], are attributed to six carbon atoms of the glucose unit. However, there is no signal of C-4 and C-6 of amorphous cellulose in the spectrum, suggesting the complete disruption of the cellulose amorphous structure during the acid hydrolysis of cotton. Notably, two more intense signals appear in the spectrum of fiber wood modified (Fig. 3) in addition to those of fiber wood, due to the carbon atoms of carboxylic groups $\mathrm{C}-7$ at $173.8 \mathrm{ppm}$ and methylene carbon in the alkyl moiety at 20-40 ppm.

\section{Contact angle measurement}

By analyzing the result concerning the evolution of contact angles as a function of time, a clear difference can be seen in the variation of these angles (Fig. 4). In fact, the alkaline treatment of the fibers with sodium hydroxide increases the hydrophilic appearance, and reveals the contact angle recorded for this sample, which remained stable around $20^{\circ}$. The same is noticed from the fibers handled by soda/EDTA, the contact angle varies from $45^{\circ}$ at the beginning. From the obtained curves, we notice that the wood modified by grafting of hydrocarbon chain possesses the highest angle $\left(70^{\circ}\right)$, it suggests that the evolution of character of surface is bound to the presence of chain alkyls which are going to paper the surface while adopting a perpendicular configuration on the surface to that this.

The value of the contact angle of a drop of water onto the surface of the grafted fibers with increasing level of esterification is depicted in Fig. 5. Results show that the angle $\theta$ increased with increasing aliphatic chain length and the esterification extent. For modified wood fibers having an acylation degree higher then DS $=0.43, \theta$ exceeded $90^{\circ}$, indicating that the surface became totally hydrophobic.

\section{Recovery of pores}

The hydration of cement favors the liberation of a very important quantity of calcium ions, the addition of a low concentration by adjuvant anionic (anionic surfactant SDBS)
Fig. 2 FTIR spectra of wood fiber (WF) and modified with $\mathrm{C} 12$ anhydride at $\mathrm{DS}=0.43$

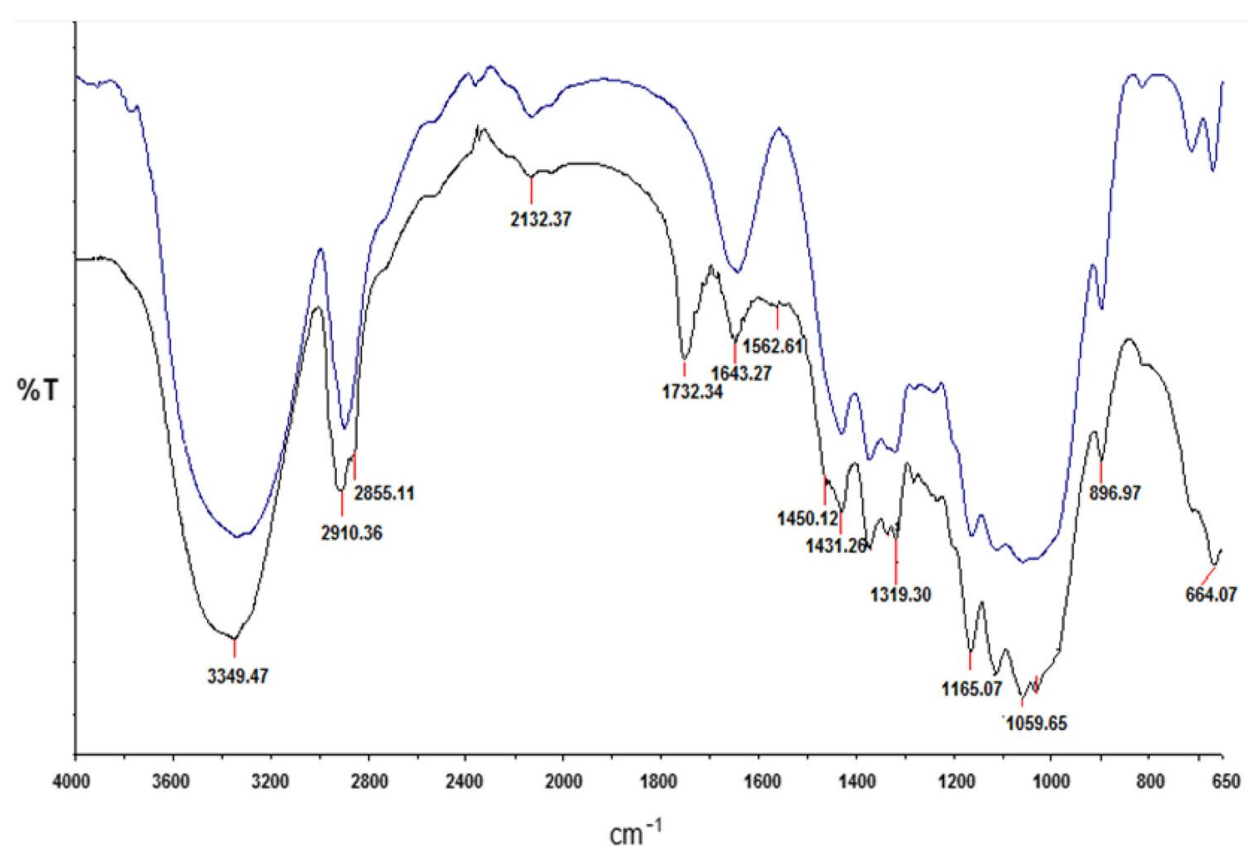




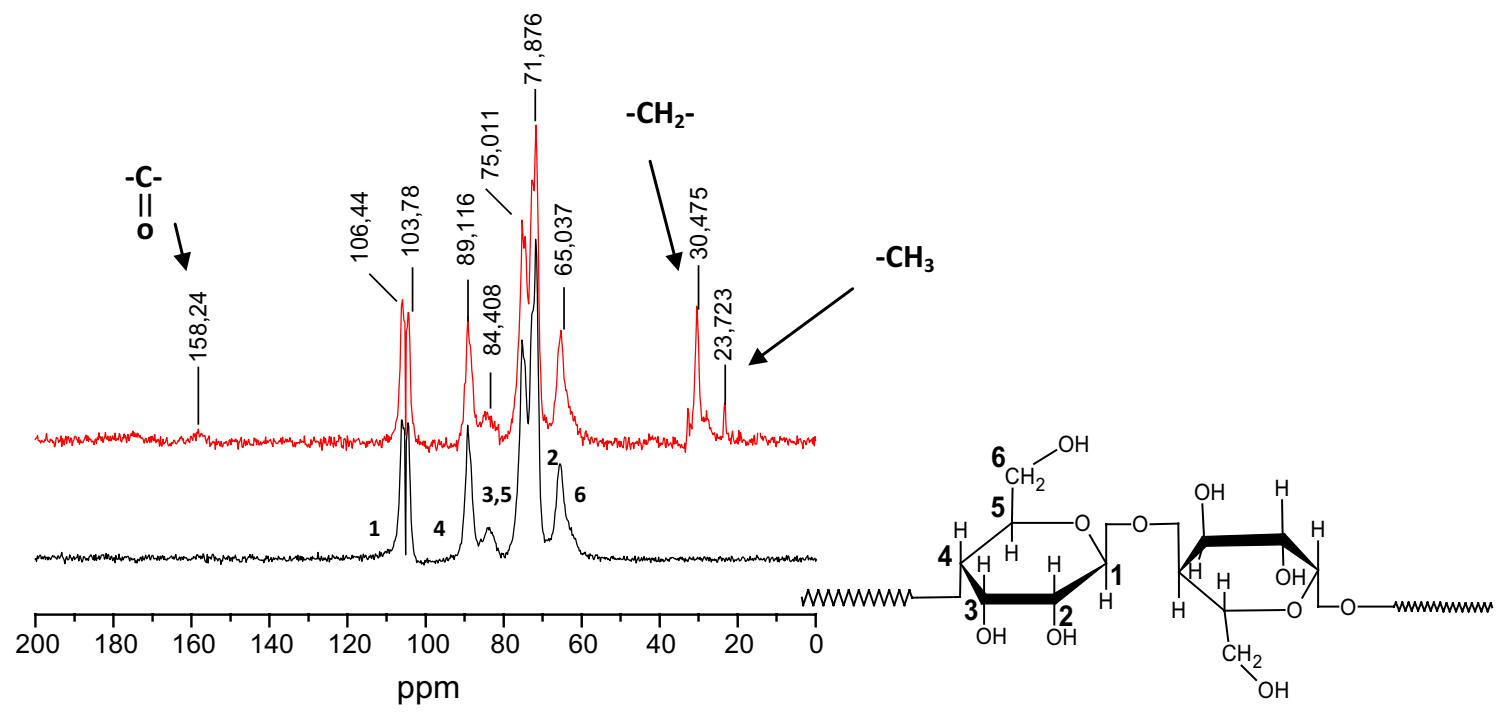

Fig. 3 CP-MAS 13CNMR spectra of wood fiber (WF) and the modified after esterification

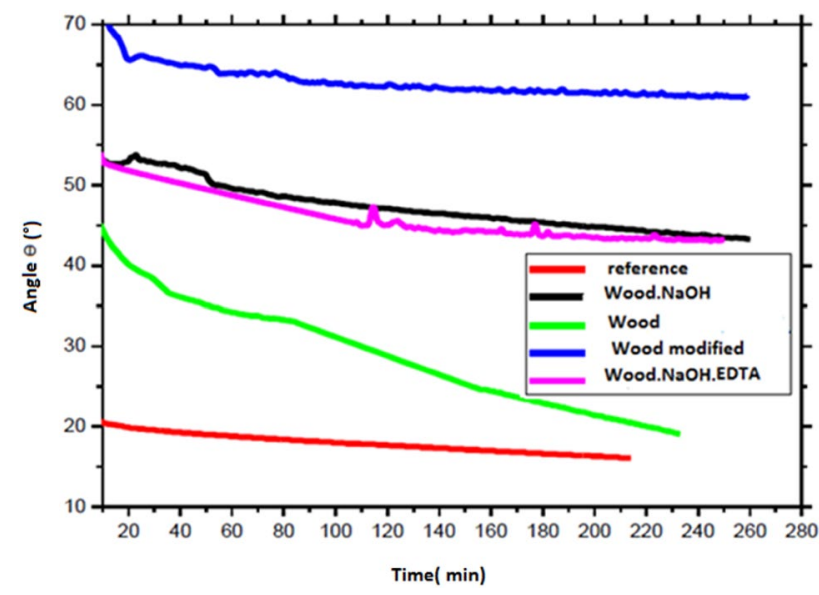

Fig. 4 Variation of the contact angle as a function of the fiber treatment

can assure the electrostatic neutralization. If we add a higher concentration of this additive, we shall have in this case an excess of the molecules of SDBS in the mixture. By arriving at the CMC (critical micellar concentration), the molecules of the additive group together by forming micelles responsible for the superficial cover of complex $\mathrm{Ca}^{2+} /$ pectin, and as a result of recovery of pores in composites.

\section{Electrokinetic study of the fiber wood suspensions handled by SDBS in the presence of a cement matrix}

Every surfactant possesses a certain concentration molar called "critical micellar concentration" (CMC), from which it will be capable of forming micelles grouping together on the superficial part of the composite. This operation gives

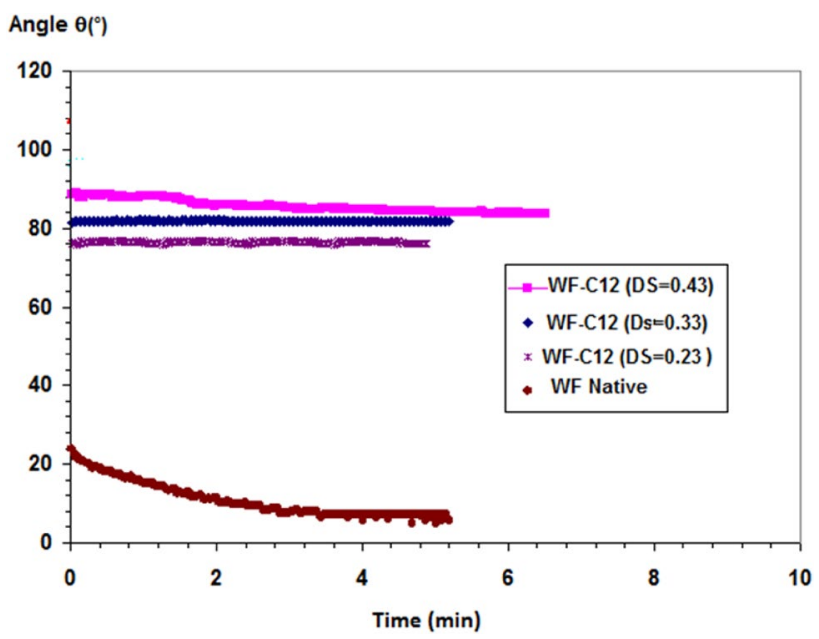

Fig. 5 Evolution of the surface of the composite (MCP) material from hydrophilic state (with wood fiber: unmodified WF) to the hydrophobic state (with modified wood fiber for $\mathrm{DS}=0 ; 0.23 ; 0.33$; $0.43)$

birth to a phenomenon of emulsion (Fig. 6). The addition of an anionic additive (SDBS) in the water by mixing improves of more the surface of samples by minimizing the size of pores by emulsifying effect, from where the absorption of water reduces (Fig. 7).

According to the curve of the potential Zeta, we notice that the addition of the additive in the manufacturing of composites, pass by several stages. Indeed, for a low concentration of the $\mathrm{SDBS} \sim\left(0.1 \mathrm{mmol} \mathrm{L}^{-1}\right)$, the potential Zeta is of the order of $35 \mathrm{mV}$, this value results from the ionization of calcium during the hydration of cement. The more the concentration by adjuvant increases, the more 
Fig. 6 State of the environment of cement grains: emulsion

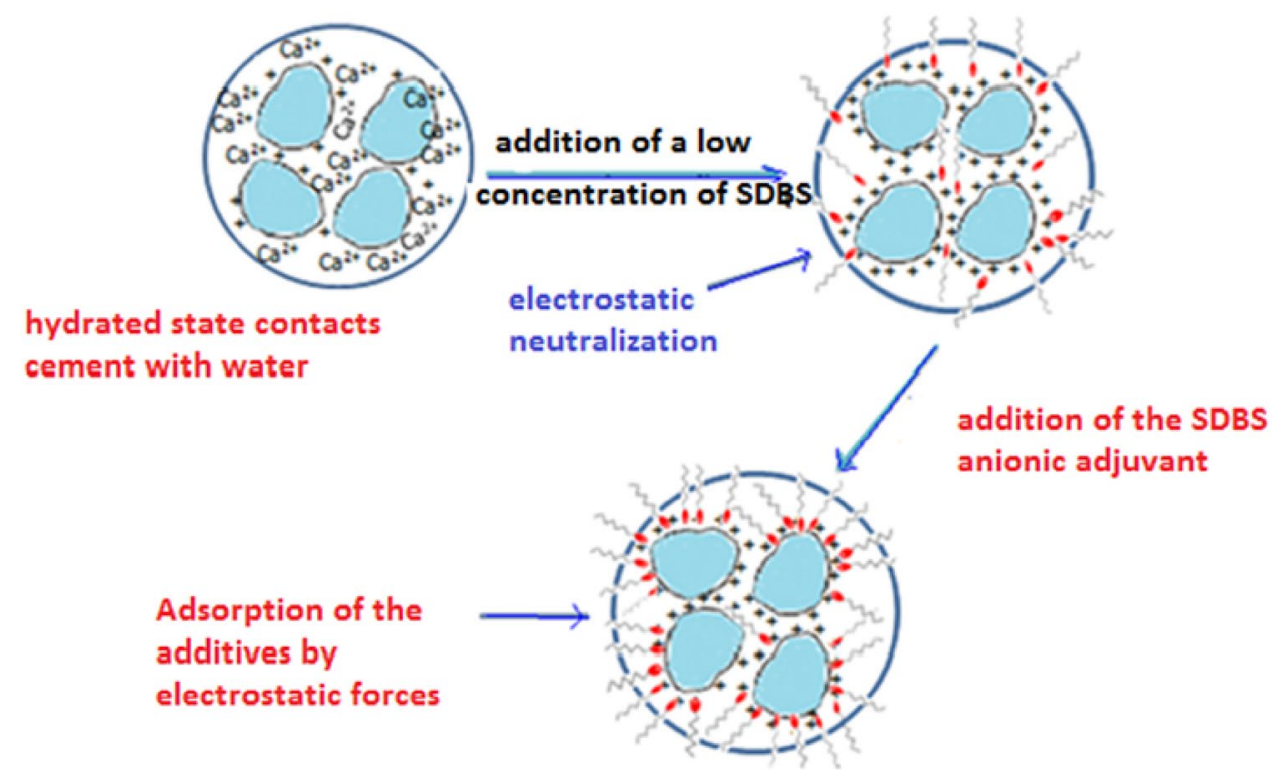

Adsorption of the additives by electrostatic forces neutralization addition of the SDBS anionic adjuvant
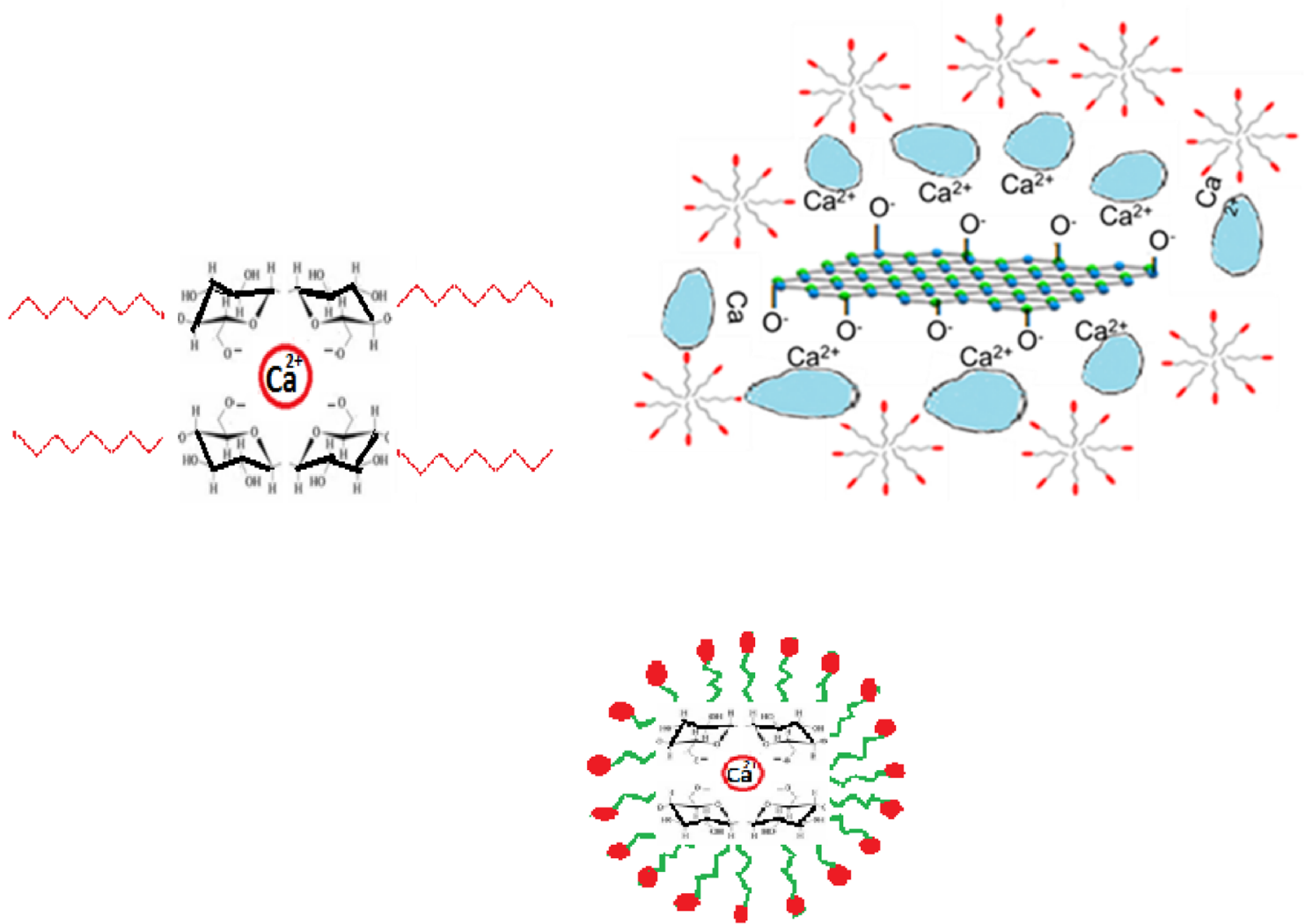

Fig. 7 Formation of micelles around wood fibers

the potential Zeta decreases; this relation informs us about the role of the molecules of the anionic additive in the neutralization of the ions $\mathrm{Ca}^{2+}$. By arriving at a certain concentration of the additive $\sim\left(1.1 \mathrm{mmol} \mathrm{L}^{-1}\right)$, the total neutralization was made and we have negative values of the potential Zeta. These show that the molecules of the additive are in excess, where from the formation of micelles from the CMC (Fig. 8). 
Fig. 8 Variation of the zeta potential as a function of the concentration of adjuvant SDBS

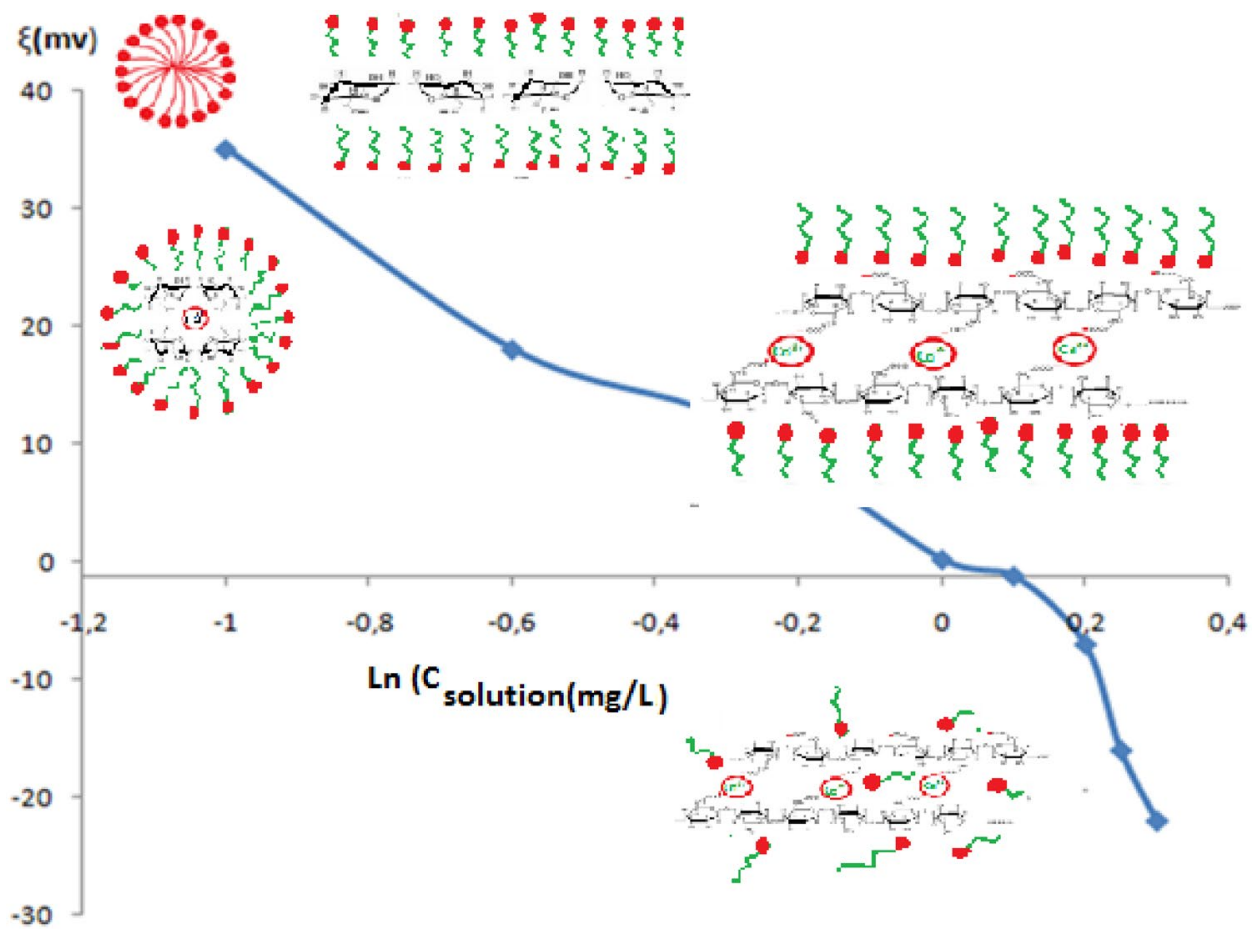

\section{Characterization physical appearance}

In this part, we have solved one of the major concerns about our subject, namely the problem of water absorption. This last factor is responsible for the decarbonization of steels in cementitious materials and therefore negatively affects their life expectancy.

\section{Absorbance in water}

*Effect of the treatment processing of the wood on the absorbance: the results obtained in Fig. 9 show that treatments processing made on wood fibers have certain effects on the absorbance in water of samples.

*Alkaline treatment: the dumping of fibers in a basic solution of $\mathrm{NaOH}$ can engender the ionization of the group hydroxylate $\mathrm{OH}$ on the surface of fibers [26]:

$($ Fiber $-\mathrm{OH})+\mathrm{NaOH} \rightarrow\left(\right.$ Fiber $\left.-\mathrm{O}^{-}+\mathrm{Na}^{+}\right)+\mathrm{H}_{2} \mathrm{O}$.

This anionic structure of fibers favors the collision of water molecules through electrostatic interactions. This justifies then the fact that the wood handled by the present soda a rate of absorbance raised with regard to compared with the only cement (Fig. 10).

*Treatment processing by the EDTA: concerning the chemical interaction fibers-matrices, wood fibers seem

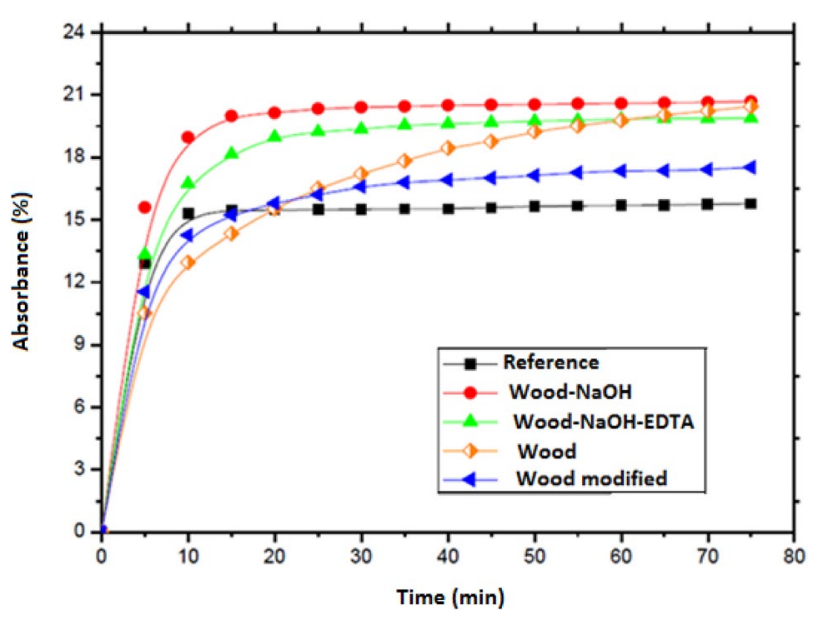

Fig. 9 Effect of fiber treatment on water absorbency

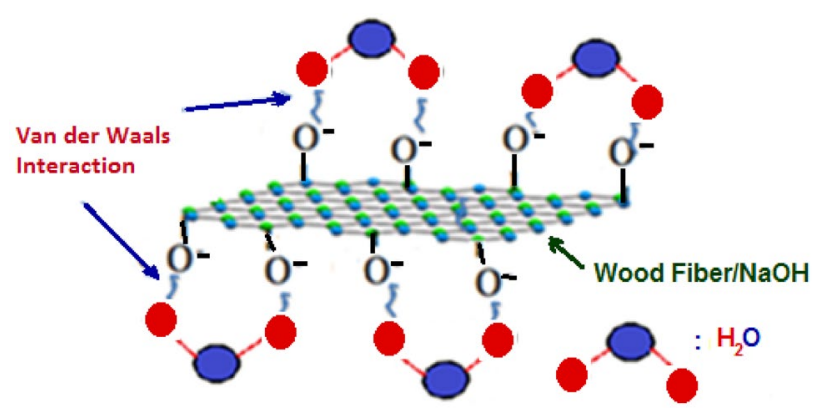

Fig. 10 Effect of the alkaline treatment processing on fibers 


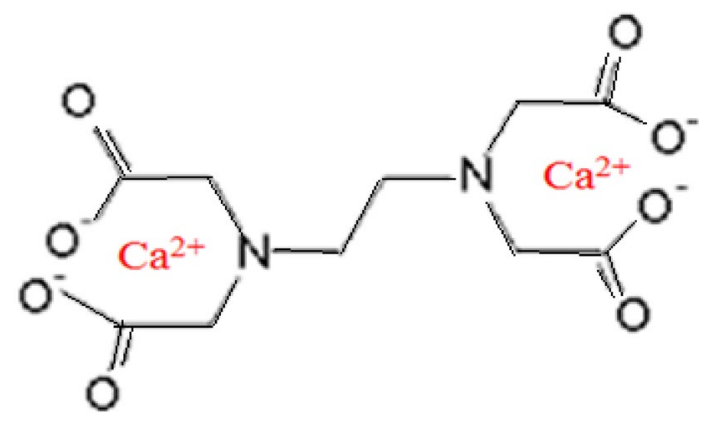

Fig. 11 Complex to $\mathrm{Ca}^{2+} /$ EDTA (effective chelate)

capable of getting an important quantity of calcium on their surfaces. This fixation of the ions $\mathrm{Ca}^{2+}$ by pectin inhibits the growth of silicate of calcium hydrated $(\mathrm{C}-\mathrm{S}-\mathrm{H})$, and is probably responsible for delays of taking observed in the dough of cement (Fig. 11). Give a complex to $\mathrm{Ca}^{2+} /$ EDTA (effective chelate) with the aim of solving this problem; we tried to prevent the fixation of the ions $\mathrm{Ca}^{2+}$ on the surface of fibers by a treatment processing in the EDTA. In fact, the EDTA by its strong chelating power (or having complexes) can train (form) a very stable complex with the calcium ions, where from we can say that this substance contributes to the destruction of complex $\mathrm{Ca}^{2+} /$ pectin.

*Treatment processing by grafting of alkyl chains: From a measurement of absorbance, we noticed that the linear hydrocarbon chains transplanted on wood fibers were the most effective with regard to the other treatments processing. The decrease of the content of water absorbed for the sample strengthened by modifying fibers, based on the role of chains alkyls which substitute the groupings hydroxyls on the surfaces of wood fibers. These chains thus inhibit the fixation of an important quantity of water on fibers (Fig. 12).

**Effect of the adjuvant SDBS: In this part, we added an anionic additive, SDBS (sodium dodecylbenzene sulfonates), to study its effect on the surface of samples and as a consequence on the absorbance in water. The realized measures showed that the contribution of the additive is

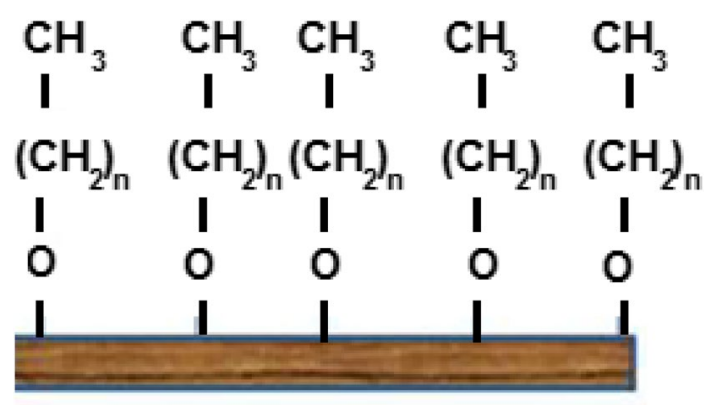

Fig. 12 Alkyl chains transplanted on the surface of fibers

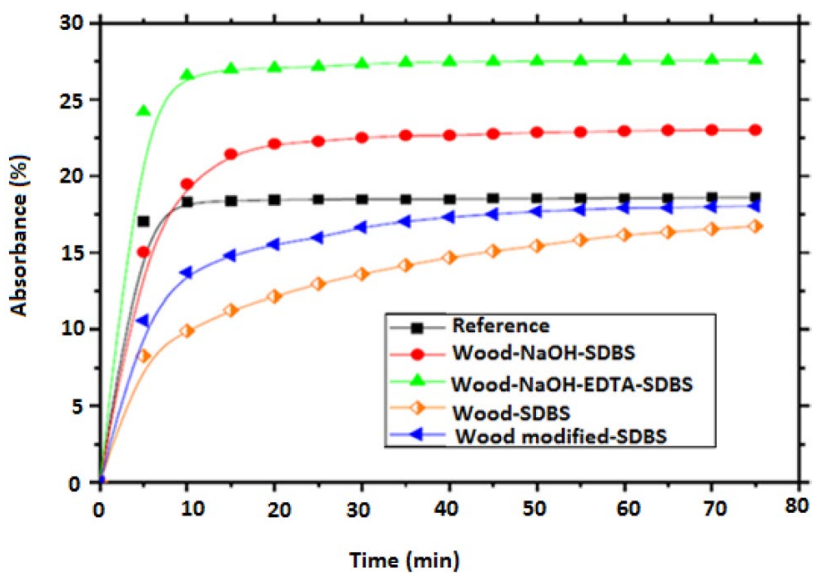

Fig. 13 Effect of the adjuvant on water absorbance

very remarkable in the reduction of the quantity of absorbed water (Fig. 13). The decrease of the rate of water absorbed by test tubes containing modified fibers and gross fibers about $20 \%$ and $35 \%$, respectively, with regard to the reference highlights the role of the additive in the superficial packaging of fibers. This result can be interpreted by the fact that the molecules of the used additive work as a superplasticizing which covers the surface of fibers preventing as a consequence their contact with the water. The reactions between the various constituents of the sample containing modified fibers can be realized as indicated in Fig. 14. The molecules of the additive play the role of an intermediate element between the ions of calcium stemming from the hydration of the cement on one hand, and the alkyl chains transplanted on the surface of fibers on the other hand. They are capable of getting a significant number of $\mathrm{Ca}^{2+}$ by their hydrophilic grouping through electrostatic connections, leaving their hydrophobic part bound, by connections of hydrophobic type, in the hydrocarbon chains. The decrease in the rate of absorption, thus results from the reduction of pores within the mud samples. What justifies the superficial shine of these test tubes when one we use the additive.

**Effect of the rate of reinforcement: For this part, we made five cement-based samples white and with some wood

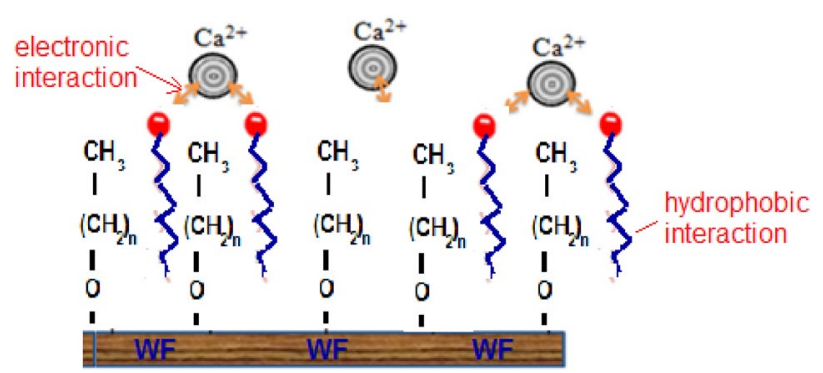

Fig. 14 Effect of the adjuvant (SDBS) on the interface fiber/matrix 


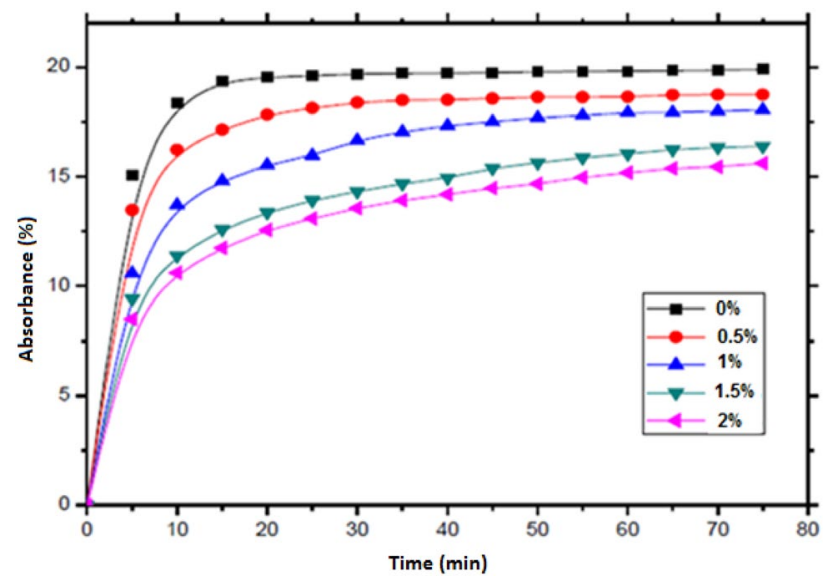

Fig. 15 Effect of the rate of reinforcement on the absorbance

modified with various rates $(0 ; 0.5 ; 1 ; 1.5$ and $2 \%)$, by adding a small quantity of additive to the water by mixing (Fig. 15). The measures of the absorbance showed an inversely proportional relation between the quantity of absorbed water and the percentage of wood fiber injected in the dough of cement. Indeed, if the rate of fibers increases, the content of absorbed water is reduced. For example, $2 \%$ of fibers are capable of minimizing the quantity of water absorbed about $21 \%$ to compare with a test tube containing $0.5 \%$ of the same wood. The relation between the rate of fibers and the absorbance is can be due to the phenomenon of emulsion which will be more intense by increasing the density of fibers.

\section{Mechanical characterization}

\section{Compressive strength}

The analyses of Fig. 16 examine the decrease in compressive stress with the increase in the percentage of fibers in cement. With the addition of $1 \%$ of the fibers, there is a decrease in

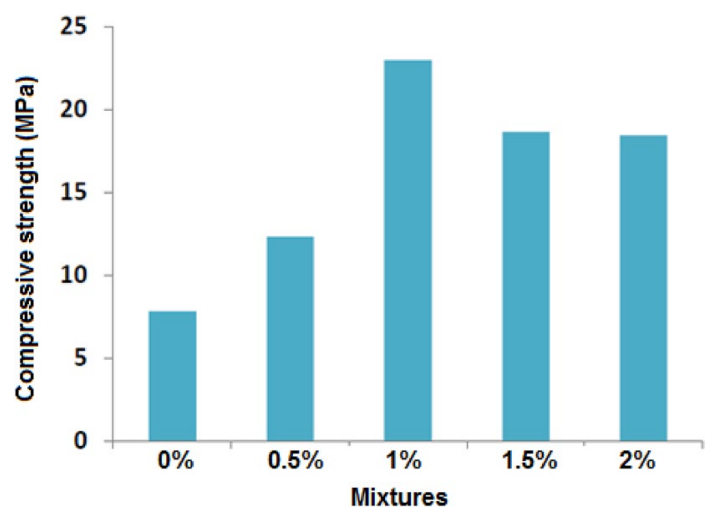

Fig. 16 The compressive strenghts of modified wood fiber-reinforced cementt pastes (after 28 days of curing) stress up to $12.33 \mathrm{MPa}$. By exceeding the addition of $2 \%$ of the fibers, no effect is observed on the compressive strength (stress) and in this case the incorporation of the fibers in the matrix increases the vacuum and reduces the compactness during the audition. The influence of the incorporation of the wood fiber leads to a constant increase in the compression force up to a content of $1 \%$ by weight. For example, the compressive strength goes from $9.81 \mathrm{MPa}$ for the pure reference cement to about $22.96 \mathrm{MPa}$, which corresponds to an improvement of about $200 \%$. However, at $2 \%$ by weight of wood fiber, the concentration falls considerably below that of the pure cement. Three possible reasons have been put forward for the considerable improvement of the mechanical properties with the addition of wood fiber in the cement. First, because of their hydrophilic nature, hydration of the cement is accelerated and larger volumes of hydration products are formed. Second, the high surface area of wood fiber enhances the fiber-matrix interface, which improves the transfer of stress between the matrix and the fibers. Third, the high ratio of surface to wood fiber volume has favored the nucleation of the cement phases, namely $\mathrm{CSH}$, providing excellent chemical reactivity to promote hydration of the cement. Finally, the application of the addition of SDBS, as a dispersing agent, also contributed to the improvement of the resistance: This improves the dispersion of wood fiber in the cement matrix. The carboxylic groups on the wood fiber surface react with the hydrated calcium silicate and result in a strong coating force between the fiber and the matrix. This reaction can provide a higher stress when the crack goes directly to the zone of the reinforced wood fiber zone and prevents the propagation of cracks. Furthermore, when the wood fiber reacts chemically with hydration products, they can behave as nucleation and form interfacial mechanical bonds. More than $1 \mathrm{wt} \%$ of wood fiber, a possible reason for the decrease in resistance to be due to aggregation of wood fiber, leading to inhomogeneous dispersion of the wood fiber in the cement matrix. This agglomeration is attributed to locating high wood fiber content and forming networks. These agglomerated wood fibers create fragile areas in the form of pores. After the curing process, these particles act as stress concentrators in the cement matrix and promote premature cracking. The cement reinforcement hypothesis can be mainly attributed to the wood fiber potential to improve the degree of hydration of the cement.

\section{Young's modulus " $E$ "}

The presence of wood fibers in a cement matrix confirms the inrease in rigidity, which is explained by the inrease of the Young's modulus at $1 \%$ of fiber addition, which increases the stress and the material also becomes more resistant. We note in Table 3 that the materials had a better maximum stress on the order of $22.96 \mathrm{MPa}$ for $1 \%$ of the fibers treated 
Table 3 Young's modulus values of the different samples

\begin{tabular}{lcc}
\hline Sample & $E(\mathrm{GPa})$ & $\begin{array}{l}\text { Compressive } \\
\text { strength (MPa) }\end{array}$ \\
\hline Cement $+0 \%$ wood modified & 57 & 9.81 \\
Cement $+2 \%$ wood modified & 476 & 18.42 \\
Cement $+1.5 \%$ wood modified & 69 & 18.62 \\
Cement $+1 \%$ wood modified & 101 & 22.96 \\
Cement $+0.5 \%$ wood modified & 58 & 12.33 \\
\hline
\end{tabular}

with SDBS. The presence of fibers wood improves the rupture strength of composite cement; this expression was confirmed as well by the obtained results.

\section{Conclusion}

In this work, the fiber wood modified potential, as reinforcement for a cementitious matrix, was investigated. Due to its hydrophilic potental, high reactivity and high-specific surface area: the addition of modified wood fiber has shown an improvement in the mechanical and microstructural properties aoàf the new composite: modified wood fiber-portland cement.

The experimental results have shown that the incorporation of the fiber wood modified has greatly enhanced the compressive strength. The highest strength property was observed by adding $1 \mathrm{wt} \%$ of the fiber wood modified. On the other hand, these samples showed the effectiveness of the grafting of the hydrocarbon chains on the surface of the fibers, in the reduction of the absorbed rate in water compared with only cement. This feature has been further improved by adding to the composite in the fresh state an amount of an anionic additive (SDBS).

Open Access This article is distributed under the terms of the Creative Commons Attribution 4.0 International License (http://creativeco mmons.org/licenses/by/4.0/), which permits unrestricted use, distribution, and reproduction in any medium, provided you give appropriate credit to the original author(s) and the source, provide a link to the Creative Commons license, and indicate if changes were made.

\section{References}

1. Faruk O, Bledzki AK, Fink HP, Sain M (2012) Biocomposites reinforced with natural fibers: 2000-2010. Prog Polym Sci 37(11):1552-1596

2. Tonoli GHD, Santos SF, Savastano H, Delvasto S, de Gutiérrez RM, de Murphy MDML (2011) Effects of natural weathering on microstructure and mineral composition of cementitious roofing tiles reinforced with fique fibre. Cem Concr Compos 33(2):225-232
3. Toledo RDF, Fairbain EMR, Filho JAM, Silva FA (2009) Durability of compression molded sisal fiber reinforced mortar laminates. Constr Build Mater 23:2409-2420

4. Olivito RS, Cevallos OA, Carrozzini A (2014) Development of durable cementitious composites using sisal and flax fabrics for reinforcement of masonry structures. Mater Des 57:258-268

5. Tolëdo FRD, Ghavami K, Englan DGL, Scrivener K (2003) Development of vegetable fibers-mortar composites of improved durability. Cem Concr Compos 25:12

6. Reis JML (2006) Fracture and flexural characterization of natural fiber-reinforced polymerconcrete. Constr Build Mater 20(9):673-678

7. Zhou F, Cheng G, Jiang BO (2014) Effect of silane treatment on microstructure of sisal fibers. Appl Surf Sci 292:806-812

8. Boustingorry P, Grosseau P, Guyonnet R, Guilhot B (2005) The influence of wood aqueous extractives on the hydration kinetics of plaster. Cem Concr Res 35(11):2081-2086

9. Ipavec A, Gabrovsek R, Vuk T, Kaucic V, Macek J, Meden A (2011) Carboaluminate phases formation during the hydration of calcite-containing Portland cement. J Am Ceram Soc 94:1238-1242

10. Toledo Filho RD, Scrivener K, England GL, Ghavami K (2000) Durability of alkali-sensitive sisal and coconut fibres in cement mortar composites. Cem Concr Compos 22(2):127-143

11. Thomason JL, Carruthers J, Kelly J, Johnson G (2011) Fibre cross-section determination and variability in sisal and flax and its effects on fibre performance characterization. Compos Sci Technol 71(7):1008-1015

12. Coutts RS, Ward JV (1987) Microstructure of wood-fibre-plaster composites. J Mater Sci Lett 229(5):562-564

13. Antonio NN, Hiroyuki Y (2008) The effect of fiber content on the mechanical and thermal expansion properties of biocomposites based on microfibrillated cellulose. Cellulose 15:555-559

14. Bayoumi TA, Saleh HM (2018) Characterization of biological waste stabilized by cement during immersion in aqueous media to develop disposal strategies for phytomediated radioactive waste. Prog Nucl Energy 107(8):83-89

15. Saleh HM, Eskander SB (2009) Long-term effect on the solidified degraded cellulose-based waste slurry in cement matrix. Acta Montan Slovaca 14(4):291-297

16. Saleh HM, Tawfik ME, Bayoumi TA (2011) Chemical stability of seven years aged cement-PET composite waste form containing radioactive borate waste simulates. J Nucl Mater 411(1-3): 185-192

17. Saleh HM, Eskander SB (2012) Characterizations of mortardegraded spinney waste composite nominated as solidifying agent for radwastes due to immersion processes. J Nucl Mater 430(1-3):106-113

18. Saleh HM (2014) Stability of cemented dried water hyacinth used for biosorption of radionuclides under various circumstances. J Nucl Mater 446(1-3):124-133

19. Saleh HM, Eskander SB, Fahmy HM (2014) Mortar composite based on wet oxidative degraded cellulosic spinney waste fibers. Int J Environ Sci Technol 11(5):1297-1304

20. Eskander SB, Saleh HM, Fahmy HM (2009) Incorporation of the spinning wastes in cement and mortars. J Radiat Res Appl Sci 2(1):119-136

21. Eskander SB, Bayoumi TA, Saleh HM (2012) Performance of aged cement-polymer composite immobilizing borate waste simulates during flooding scenarios. J Nucl Mater 420(1-3):175-181

22. Eskander SB, Saleh HM (2012) Cement mortar-degraded spinney waste composite as a matrix for immobilizing some low and intermediate level radioactive wastes: consistency under frost attack. J Nucl Mater 420(1-3):491-496 
23. Eskander SB, Bayoumi TA, Saleh HM (2013) Leaching behavior of cement-natural clay composite incorporating real spent radioactive liquid scintillator. Prog Nucl Energy 67:1-6

24. Aloulou F, Boufi S, Belgacem N, Gandini A (2004) Adsorption of cationic surfactants and subsequent adsolubilization of organic compounds onto cellulosic fibres. Colloid Polym Sci 283:344-350

25. Aloulou F, Boufi S, Beneventi D (2004) Adsorption of organic compound onto polyelectrolyte immobilized-surfactant aggregates onto cellulosic fibres. J Colloidal Interface Sci 280(2):350-358

26. Aloulou F, Boufi S, Chakchouk M (2004) Adsorption of ocatdecyltrimethylammonium chloride and adsolubilization onto cellulosic fibres. Colloid Polym Sci 282(7):699-707

27. Aloulou F, Boufi S, Labidi J (2006) Modified cellulose fibres for adsorption of organic compound in aqueous solution. Sep Purif Technol 52:332-342

28. Alila S, Aloulou F, Beneventi D, Boufi S (2007) Self-aggregation of cationic surfactants onto oxidized cellulose fibres and coadsorption of organic compounds. Langmuir 23:3723-3731

29. Alila S, Boufi S, Belgacem N, Beneventi D (2005) Adsorption of cationic surfactant onto cellulose fibres I; surface charge effects. Langmuir 21:8106-8113
30. NT 47.13 (1983): liants hydrauliques-Techniques des essais- essais de retrait de gonflement, Journal officiel de la rébublique Tunisienne $\mathrm{N}^{\circ} 70$, pp 3178

31. European standard EN 197-1, "Cement-Part 1 composition, specifications and conformity criteria for common cements", Prepared by Technical committee CEN/ T C 51, 2000

32. NT 47.30:1991.'Méthode d'essais des ciments: Détermination des résistances mécaniques', Journal officiel de la République Tunisienne, $\mathrm{N}^{\circ} 70$, pp 3178

33. Liu H, Sale KL, Holmes BM, Simmons BA, Ande Singh S (2010) Understanding the interactions of cellulose with ionic liquids: a molecular dynamics study. J Phys chem B 114(12):4293-4301

Publisher's Note Springer Nature remains neutral with regard to jurisdictional claims in published maps and institutional affiliations. 